\title{
NS5A Gene Analysis by Next Generation Sequencing in HCV Nosocomial Transmission Clusters of HCV Genotype 1b Infected Patients
}

\author{
Maria Concetta Bellocchi ${ }^{1, \dagger}{ }^{\dagger}$, Marianna Aragri ${ }^{1,+}{ }^{,}$Luca Carioti ${ }^{1}$, Lavinia Fabeni ${ }^{1}$, \\ Rosaria Maria Pipitone ${ }^{2}$, Giuseppina Brancaccio ${ }^{3,4}$, Maria Chiara Sorbo ${ }^{1}{ }^{1}$, Silvia Barbaliscia ${ }^{1}$, \\ Velia Chiara Di Maio ${ }^{1}$, Fabrizio Bronte ${ }^{2}$, Stefania Grimaudo ${ }^{2}$, Walter Mazzucco ${ }^{2} \mathbb{D}$, \\ Ferdinando Frigeri ${ }^{5}$, Marco Cantone ${ }^{3}$, Antonio Pinto ${ }^{6}$, Carlo Federico Perno ${ }^{7}$, Antonio Craxì ${ }^{2}$, \\ Giovanni Battista Gaeta ${ }^{3}$, Vito Di Marco ${ }^{2}$ D and Francesca Ceccherini-Silberstein $^{1, *}$ \\ 1 Department of Experimental Medicine, University of Rome Tor Vergata, 00133 Rome, Italy \\ 2 Health Promotion Sciences, Maternal and Infant Care, Internal Medicine and Medical Specialties (PROMISE) \\ Department, University of Palermo, 90127 Palermo, Italy \\ 3 Infectious Diseases, Department of Mental and Physical Health and Preventive Medicine, Campania \\ University “Luigi Vanvitelli”, 80138 Naples, Italy \\ 4 Department of Molecular Medicine, University of Padua, 35121 Padua, Italy \\ 5 Hematology Division, "San Sebastiano" Hospital, 81100 Caserta, Italy \\ 6 Hematology Department, National Cancer Institute "Fondazione Pascale", IRCCS, 80131 Naples, Italy \\ 7 Department of Microbiology and Clinic Microbiology, University of Milan, 20162 Milan, Italy \\ * Correspondence: ceccherini@med.uniroma2.it; Tel.: +0039-0672-596-566 \\ + These authors contributed equally to this work.
}

Received: 9 April 2019; Accepted: 25 June 2019; Published: 2 July 2019

\begin{abstract}
Background: The aim of the study was to investigate the intra-host variability through next-generation-sequencing (NGS) of the NS5A-gene in nosocomial transmission-clusters observed in two Italian hospitals among hepatitis $\mathrm{C}$ virus (HCV)-genotype-1b infected patients. Methods: HCV-sequencing was performed by Sanger-sequencing (NS3 + NS5A + NS5B) and by NGS (NS5A, MiSeq-Illumina) in $15 \mathrm{HCV}-1 \mathrm{~b}$ infected patients [five acute with onco-hematologic-disease and 10 (4/6 acute/chronic) with $\beta$-thalassemia]. Resistance-associated-substitutions (RAS) were analysed by Geno2pheno-algorithm. Nucleotide-sequence-variability (NSV, at 1\%, 2\%, 5\%, $10 \%$ and 15\% NGS-cutoffs) and Shannon entropy were estimated. Phylogenetic analysis was performed by Mega6-software and Bayesian-analysis. Results: Phylogenetic analysis showed five transmission-clusters: one involving four $\mathrm{HCV}$-acute onco-hematologic-patients; one involving three HCV-chronic $\beta$-thalassemia-patients and three involving both $\mathrm{HCV}$-acute and chronic $\beta$-thalassemia-patients. The NS5A-RAS Y93H was found in seven patients, distributed differently among chronic/acute patients involved in the same transmission-clusters, independently from the host-genetic IL-28-polymorphism. The intra-host NSV was higher in chronic-patients versus acute-patients, at all cutoffs analyzed $(\mathrm{p}<0.05)$. Even though Shannon-entropy was higher in chronic-patients, significantly higher values were observed only in chronic $\beta$-thalassemia-patients versus acute $\beta$-thalassemia-patients $(p=0.01)$. Conclusions: In nosocomial HCV transmission-clusters, the intra-host $\mathrm{HCV}$ quasispecies divergence in patients with acute-infection was very low in comparison to that in chronic-infection. The NS5A-RAS Y93H was often transmitted and distributed differently within the same transmission-clusters, independently from the IL-28-polymorphism.
\end{abstract}

Keywords: HCV; acute infection; chronic infection; nosocomial transmission; sequencing; NGS 


\section{Introduction}

Hepatitis $\mathrm{C}$ is a liver disease caused by the hepatitis $\mathrm{C}$ virus (HCV). The virus can cause both acute and chronic hepatitis. Globally, an estimated 71 million people have chronic hepatitis $C$ infection [1]. Acute HCV infection is usually defined as having an estimated duration of infection of less than six months [2]. The incubation period for hepatitis $C$ is from two weeks to six months. Since acute HCV infection is usually asymptomatic, very few people are diagnosed during the acute phase. Furthermore, the majority of individuals develop chronic HCV infection having been previously undiagnosed, as the infection may remain asymptomatic for decades until symptoms slowly reveal serious liver damage [3,4]. A significant number of chronically infected individuals will develop cirrhosis or liver cancer $[5,6]$.

$\mathrm{HCV}$ is mainly a bloodborne virus, commonly transmitted through injecting drugs, reuse or inadequate sterilization of medical equipment, or the transfusion of unscreened blood and blood products. Cases of HCV transmission in healthcare settings have been reported [7-9]. However, reports on transmissions during acute infection diagnosis are still limited [10].

At present, HCV is classified into eight major genotypes and at least 100 subtypes [11-13]. The worldwide distribution and prevalence of each HCV genotype (GT) vary depending on geographical region. A recent global survey found that GT 1 (subtypes 1a and 1b predominate in Europe, North America, and Australia) and GT 3 (primarily found in South Asia, particularly the Indian sub-continent) are the most prevalent, accounting for $46 \%$ and $30 \%$ of all infections, respectively. The GT 2 (prevalent in Western Africa), GT 4 (prevalent in the Middle East and North and Central Africa), GT 5 (prevalent in South Africa) and GT 6 (prevalent in Southeast Asia) accounted for 9\%, 8\%, 1\% and 6\%, respectively. The GT 7 (in Central Africa) and GT 8 (from the Punjab, in India) were found in a few individuals $[12,14,15]$. In Italy, in recent decades, there has been a change in the epidemiology of viral hepatitis thanks to improved hygienic and socio-economic conditions. However, the HCV epidemics in Italy is still the highest [16] among Western European countries, with GT 1 (a and b subtypes) the most prevalent (with a different prevalence $65-45 \%$ according to the human immunodeficiency virus [HIV] coinfection status: HIV-/HIV+), followed by GT 3 (about 19-36\%, HIV-/HIV+), GT 2 and GT 4 (about 8-3\% and 9-11\%, HIV-/HIV+ respectively) and finally GT 5 (about $<1 \%$ ) [17-20].

At intra-host level, HCV is genetically diverse and exists as a quasispecies defined by several closely related yet genetically distinct populations [21]. The extremely high intrinsic genetic variability of $\mathrm{HCV}$ is a consequence of its high mutational rate and replication turnover, along with a lack of proof-reading activity of viral polymerase. As a result of this phenomenon, a natural emergence of resistance associated variants (RAVs) against direct acting antivirals (DAA) can arise and may compromise treatment [22]. This is a particular characteristic of HCV [23], although less shared by other viruses responsible for chronic infections, such as hepatitis B virus (HBV) or HIV-1, which are more associated with resistance transmission rather than natural resistance [24,25].

Host genetic factors play a crucial role in spontaneous and treatment-induced HCV infection clearance. Human single nucleotide polymorphisms (SNP) of IL28B gene (CC variant) are considered as a very good predictor of viral clearance [26-29].

Viral genomic population sequencing, combined with a phylogenetic analysis, is a very useful methodology to investigate epidemiology, evolution, resistance, outbreaks, and transmission of viruses [10,30,31]. Recent advances in the nucleic acid sequencing technologies have begun to replace population Sanger sequencing both for research purposes and in the clinical routine, also for drug resistance testing [32]. Next-Generation Sequencing (NGS) allows for accurate characterization of intra-host HCV populations, detecting low frequency variants and rare mutations [33].

Since the whole genome analysis is expensive and highly complex, the sequencing of specific HCV genomic regions, like Core and/or NS5B, represented, and still represents a more convenient method to study HCV genetic diversity [34]. Furthermore, various studies have showed that performing the sequencing of NS3 and NS5A non-structural genes involved in DAA drug resistance also produces 
highly reliable genotype information and can therefore provide both resistance and evolutionary information for molecular epidemiology $[9,10,34,35]$.

By performing phylogenetic analysis on NS3, NS5A and NS5B genes using Sanger sequencing, we recently identified separate episodes of HCV transmission in two healthcare settings: one in patients with acute $\mathrm{HCV}$ diagnosis affected by onco-hematologic disease [36] and the second in patients with acute HCV diagnosis with $\beta$-thalassemia [31]. In this present study, through NS5A NGS analysis we aimed to investigate the intra-host viral quasispecies composition and resistance transmission in 15 patients, all putatively involved in transmission clusters.

\section{Materials and Methods}

\subsection{Study Population}

Serum samples from $28 \mathrm{HCV}$ infected patients followed in two Italian hospitals were analyzed using the Sanger genotypic-resistance-test for NS3, NS5A and NS5B genes. All the patients were naïve to DAAs, with the exception of 1 patient with chronic infection who failed a DAA based regimen. Twenty-two patients were affected by $\beta$-thalassemia Major and 6 patients had an onco-hematologic disease $[31,36]$. Eleven $\beta$-thalassemia patients received a diagnosis of acute HCV infection during an outbreak between April and November 2016. Five of these received a diagnosis of de-novo acute infection documented by an increase in ALT values greater than 10 upper normal values, positive serum HCV-RNA and seroconversion from anti-HCV negative to anti-HCV positive, while 6 patients received a diagnosis of HCV reinfection since they had past evidence of HCV infection with spontaneous clearance or sustained virological response to Interferon-based therapy. Eleven $\beta$-thalassemia patients had a known chronic HCV infection documented by a persistence of serum HCV-RNA positivity. The six patients with hematological malignances received a diagnosis of de-novo acute HCV infection between January and February 2016. None of these patients had developed anti-HCV antibodies (also at the last follow-up visit), confirming their profound immunodepression state as a result of the disease and the chemotherapy received [36]. All subjects gave their informed consent for inclusion before they participated in the study. The study was conducted in accordance with the Declaration of Helsinki, and the protocol was approved by the Ethics Committee of 22 November 2018 (Project identification code: DASOE/6/prot 86605).

\subsection{HCV Sanger Sequencing}

The serum of 28 patients was tested for HCV NS3, NS5A and NS5B genotypic resistance test by standard Sanger sequencing, following the procedure described previously [37].

Resistance associated substitutions (RAS) were analysed according to Sorbo et al. Drug Resist Update 2018 [38] and Geno2pheno algorithm [39].

\subsection{HCV Next Generation Sequencing}

NS5A NGS on MiSeq platforms (Illumina Inc, San Diego, CA, USA) was performed according to Nextera XT DNA Library Kit (Illumina Inc) in 15 patients, 5 acute patients with onco-hematologic disease and 10 patients ( 4 acute; 6 chronic) with $\beta$-thalassemia.

In brief, to prepare indexed libraries from each sample for sequencing, the HCV-RNA genomic was subjected on a NS5A-RT-PCR with SuperScript III One-Step RT-PCR System (Invitrogen Life Technologies, Carlsbad, CA, USA) and when necessary on a Nested-PCR with AmpliTaq Gold DNA Polymerase (Applied Biosystems, Foster City, CA, USA). For each sample, 1 ng of amplicon was involved in a tagmentation reaction using a Nextera XT DNA Library Kit (Illumina Inc) and a unique combination of an i5 and an i7 index primer was then added to each tagmented DNA sample. The libraries were diluted at $4 \mathrm{nM}$ as a final concentration before being pooled. Finally, $15 \mathrm{pM}$ of the denatured pool was paired end sequenced using the MiSeq Nano reagent kit v2 $(2 \times 250)$ (Illumina Inc).

Detailed information is reported in Text S1. 


\subsection{Consensus Sequences at Different Cutoffs and Viral Haplotypes}

Fastq files were mapped against HCV consensus D90208 using BWA mem (available at https: //github.com/lh3/bwa) [40] then BAM files were transformed into frequency files containing the numbers of observed nucleotides (A, T, C, G, N) along NS5A gene using bamToFreq [41].

Consensus sequences and the frequency of RAS were generated and analysed with prevalence cutoffs of $1 \%, 2 \%, 5 \%, 10 \%$ and $15 \%$ using geno2pheno [ngs-freq] [42] and Geno2pheno algorithm [39], respectively.

BAM files were also used to generate viral Haplotypes using QuasiRecomb [43].

\subsection{Genetic Diversity Analysis}

Nucleotide-sequence-variability (NSV) was estimated through an ambiguous nucleotide-positions percentage in NS5A ultra-deep consensus sequences using an in-house perl script. Genetic complexity was also evaluated as amino acid variability and this parameter was used to define NS5A heterogeneity. More specifically, the Shannon entropy weighted for intra-patient prevalence of viral haplotypes (Sn) was estimated for each NS5A amino acid position for each patient. The formula is reported in the Text S2.

\subsection{Phylogenetic Analysis}

\subsubsection{Genotype Assignment}

For each patient, the HCV genotype and sub-genotype were determined. The NS3 + NS5A + NS5B sequences from Sanger sequencing were aligned and compared with reference sequences for all of the HCV genotypes and sub-genotypes (geno2pheno). The sequences were then manually edited using the Bioedit program, and gaps were removed from the final alignment. Genotype assignments were achieved by constructing phylogenetic trees, first using the Neighbor-Joining (NJ) method [44]. Distances were calculated using MEGA 6 based on the Kimura-2 parameter (K2P) model [45]. The robustness of the genotyping assignments was further tested using the Maximum Likelihood (ML) method. The ML tree was inferred using the General Time-Reversible nucleotide substitution model (GTR) with gamma-distribution among site rate heterogeneity, a proportion of invariable sites $\left(\mathrm{GTR}+\mathrm{I}+\Gamma_{5}\right)$ [46], and 1000 bootstrap replicates (Mega 6). The tree was rooted using a midpoint rooting with FigTree software v 1.4.4 (Rambaut A, Institute of Evolutionary Biology, University of Edinburgh, UK) [47].

\subsubsection{Identification of Transmission Clusters}

Transmission clusters were first deduced using a Maximum-Likelihood tree as described above by using NS5A sequences from Sanger sequencing and NGS. The robustness of the transmission clusters was further tested using a Bayesian analysis. The Bayesian phylogenetic tree was reconstructed with MrBayes [48], using a GTR $+\mathrm{I}+\Gamma_{5}$. The Monte Carlo Markov Chain (MCMC) search was run for $5 \times 10^{6}$ generations with the trees sampled every 100th generation (with a burn-in of 50\%) [49]. Statistical support was obtained by calculating the posterior probability of each monophyletic clade, and a posterior consensus tree was generated after $50 \%$ burn-in. Clades were considered epidemiological clusters only if a posterior probability $\geq 90$ was inferred.

\subsubsection{Statistical Analysis}

Statistically significant differences were calculated using Fisher's exact test and the Mann-Whitney test using R software (R Core Team, Vienna, Austria, available at: http://www.R-project.org/) [50]. 


\section{Results}

\subsection{Patient Characteristics}

This study included $28 \mathrm{HCV}$ infected patients followed in two Italian hospitals (15 males, 53.5\%) with a median age of 43 years [IQR 34-52]. For diagnostic purposes, a genotypic test based on the Sanger sequencing of NS3, NS5A and NS5B was performed. All patients were infected with GT1b. Median log serum HCV-RNA was 4.9 [IQR 3.3-6.0] IU/mL, while median [IQR] ALT and AST values (available only for the patients with acute infection) were 485 [IQR 255-718] IU/L and 271 [IQR 85-547] IU/L, respectively. The rate of IL-28B rs12979860 genotype was CC (50.0\%), CT (39.2\%) and TT (7.1\%). The clinical and virological characteristics of the 28 patients included in the study are shown in Table 1.

Table 1. Patient characteristics.

\begin{tabular}{lcc}
\hline Patients, N & $\mathbf{2 8}$ \\
\hline Patients with $\beta$-thalassemia, N & 22 \\
Patients with onco-hematologic disease, N & 6 \\
Male, N (\%) & $15(53.5)$ \\
Age, Median (IQR) & $43(34-52)$ \\
HCV genotype & $1 \mathrm{~b}(100)$ \\
Patients with available rs12979860 IL-28B genotype, N (\%) & $14(50.0)$ \\
& CC & $11(39.2)$ \\
& CT & $2(7.1)$ \\
Acute Infection, N (\%) & TT & $1(3.5)$ \\
Diagnosis of Acute Infection, Year & Unknown & $17(61)$ \\
Chronic Infection, N (\%) & 2016 \\
Naive * & & $11(39)$ \\
HCV-RNA (logIU/mL), Median (IQR) & & 27 \\
AST (IU/mL), Median (IQR) & & $4.9(3.3-6.0)$ \\
ALT (IU/mL), Median (IQR) & & $271(85-547)$ \\
\hline
\end{tabular}

* 1 patient failed a DAA-based regimen for HCV. ${ }^{* *}$ Values were calculated according to the information available (only for patients with acute infection). Abbreviations: IQR, interquartile range; IU, international unit; AST, aspartate aminotransferase; ALT, alanine aminotransferase.

\subsection{NS3-NS5A-NS5B Sanger Sequences and NS5A NGS Sequences}

Among the 28 patients analysed by Sanger sequencing, NS5B, NS3 and NS5A sequences were obtained for 23, 22 and 21 patients, respectively. The NS5A ultra-deep-sequencing was then performed in 15 patients with a positive amplification product, nine with acute infection and six with chronic infection, all putatively involved in TCs harboring HCV NS5A resistance.

The NGS yielded 4,627,078 reads with a median of 186,381 [IQR 157,296-204,495] reads per sample. For each patient, five consensus ultra-deep sequences with a prevalence cutoff of $1 \%, 2 \%, 5 \%, 10 \%$ and $15 \%$ were obtained by geno2pheno, and NS5A haplotype sequences were constructed by Quasirecomb. NGS confirmed infection with GT1b in all individuals. No cases of other genotype/subtype coinfections were present, with no additional minority non-GT1b variants, at all NGS cutoffs used.

\subsection{Cluster Identification}

To characterize the transmission clusters, a Bayesian analysis was performed in 15 patients by using both Sanger and NGS NS5A sequences. Specifically, for NGS NS5A sequences, haplotypes and five different consensus sequences (obtained at cutoff of $15 \%, 10 \%, 5 \%, 2 \%$ and $1 \%$ ) were used for each patient. Among the 15 patients analyzed, five transmission clusters involving 14 patients were identified: four onco-hematologic patients with HCV acute infection, four $\beta$-thalassemia patients with acute infection and six $\beta$-thalassemia patients with chronic infection. In onco-hematologic patients, 
only one transmission cluster was observed involving four HCV acute patients (Pt11-Pt12-Pt13-Pt15) while one patient (Pt14) was not involved in the cluster. The other transmission clusters involved patients with $\beta$-thalassemia. One cluster involved three HCV chronic patients (Pt6-Pt7-Pt9) and three transmission clusters included both acute and chronic HCV infected patients (Pt1-Pt2; Pt3-Pt4-Pt5; $\mathrm{Pt} 8-\mathrm{Pt10})$. Bayesian phylogenetic trees for the two groups of patients are shown in Figure 1.

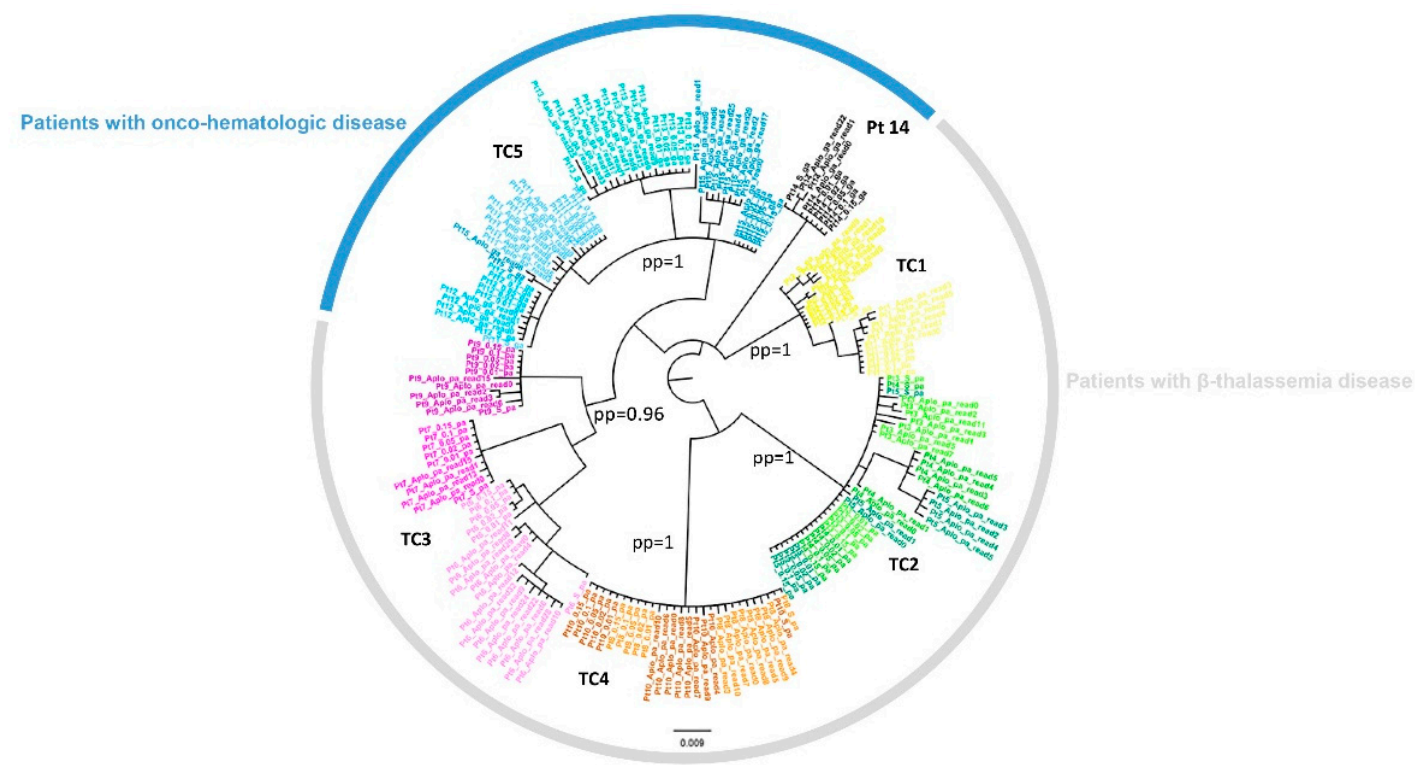

Figure 1. NS5A Bayesian Tree. The NS5A phylogenetic tree following the Bayesian method. The colors represent the different transmission clusters. Pt 14 was outside TC5. Transmission clusters (TC) were identified by a posterior probability $\geq 0.90$.

\subsection{NS3-NS5A-NS5B RASs in Sanger Sequences and in NS5A NGS Sequences}

Sanger sequencing showed no RASs in any NS3 and NS5B genes analyzed, with the exception of S122T, a polymorphism associated with resistance to NS3-inhibitors, which was found in four onco-hematologic patients, forming cluster 5. Instead, in the NS5A gene, the R30Q, L31M and/or Y93H were detected by both sequencing analyses in seven patients (Table 2).

Using NGS, no additional minority NS5A-resistant variants were identified at any of the cutoffs used. The NS5A-RAS Y93H was observed in seven out of 15 patients analyzed. Particularly, three out of nine patients $(33.3 \%)$ with acute infection and four out of six patients $(66.6 \%)$ with chronic infection presented RAS. This mutation was observed in all five transmission clusters, even if it was not shared by all the patients involved in the same cluster. In transmission cluster 2 , the $\beta$-thalassemia patient with chronic HCV infection showed the $\mathrm{Y} 93 \mathrm{H}$ at $32.9 \%$ of intra-patient prevalence, but this mutation was not present in the other two HCV acute patients, involved in the same cluster (NGS cutoff up to $1 \%$ ).

In contrast, two transmission clusters showed the same RAVs with a similar intra-patient prevalence among all individuals with both acute and chronic HCV infection. More specifically, cluster 1 showed the $\mathrm{Y} 93 \mathrm{H}$ with a prevalence $>97.8 \%$ in both $\mathrm{Pt} 1$ and $\mathrm{Pt} 2$, and cluster 4 showed a pattern of multi-resistance R30Q, L31M and Y93H with high prevalence of $>97 \%$, in both Pt8 and Pt10. Pt8 with chronic HCV infection was the only patient with a previous DAA-failure.

Finally, in cluster 5, only one HCV acute onco-hematologic-patient showed the $\mathrm{Y} 93 \mathrm{H}$ at $99.2 \%$ of intra-patient prevalence, but this mutation was not present in the other three HCV acute patients involved in the same transmission cluster (Table 2). 
Table 2. List of mutations found in each gene using Geno2pheno for both Sanger and Next-Generation Sequencing (NGS) sequences.

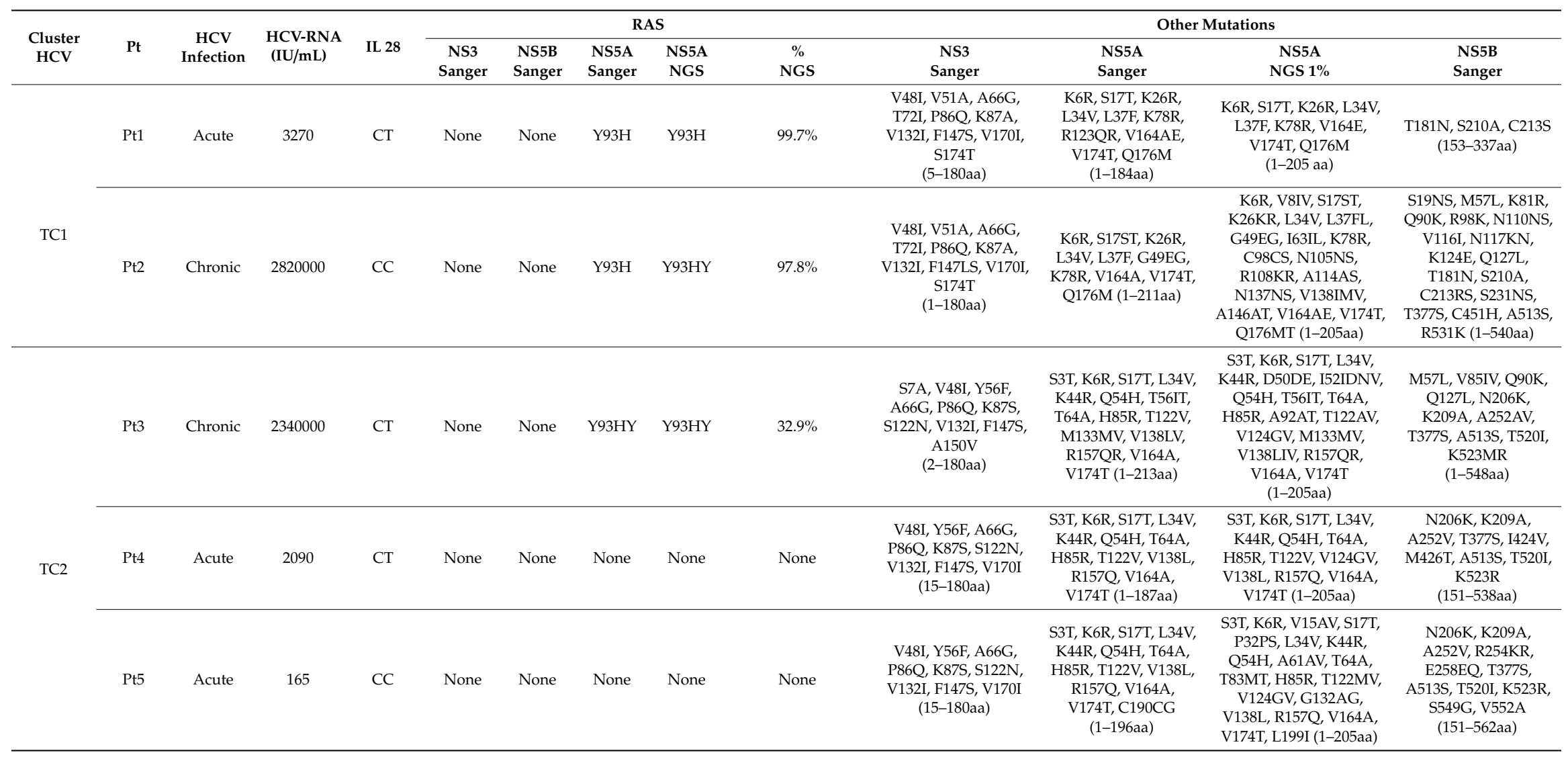


Table 2. Cont

\begin{tabular}{|c|c|c|c|c|c|c|c|c|c|c|c|c|c|}
\hline \multirow{2}{*}{$\begin{array}{l}\text { Cluster } \\
\mathrm{HCV}\end{array}$} & \multirow[b]{2}{*}{$\mathrm{Pt}$} & \multirow{2}{*}{$\begin{array}{c}\mathrm{HCV} \\
\text { Infection }\end{array}$} & \multirow{2}{*}{$\begin{array}{l}\text { HCV-RNA } \\
\text { (IU/mL) }\end{array}$} & \multirow[b]{2}{*}{ IL 28} & \multicolumn{5}{|c|}{ RAS } & \multicolumn{4}{|c|}{ Other Mutations } \\
\hline & & & & & $\begin{array}{c}\text { NS3 } \\
\text { Sanger }\end{array}$ & $\begin{array}{c}\text { NS5B } \\
\text { Sanger }\end{array}$ & $\begin{array}{c}\text { NS5A } \\
\text { Sanger }\end{array}$ & $\begin{array}{l}\text { NS5A } \\
\text { NGS }\end{array}$ & $\begin{array}{c}\% \\
\text { NGS }\end{array}$ & $\begin{array}{c}\text { NS3 } \\
\text { Sanger }\end{array}$ & $\begin{array}{l}\text { NS5A } \\
\text { Sanger }\end{array}$ & $\begin{array}{c}\text { NS5A } \\
\text { NGS 1\% } \\
\end{array}$ & $\begin{array}{l}\text { NS5B } \\
\text { Sanger }\end{array}$ \\
\hline \multirow{3}{*}{ TC3 } & Pt6 & Chronic & 1800000 & СТ & None & None & None & None & None & $\begin{array}{l}\text { S7A, C16CW, V48I, } \\
\text { S61A, A66G, P86Q, } \\
\text { K87AS, F147S } \\
\text { (1-180aa) }\end{array}$ & $\begin{array}{l}\text { K6R, S17T, L34V, } \\
\text { L37F, T56I, K78R, } \\
\text { T79A, V164A, V174T, } \\
\text { L183V, S201S, } \\
\text { M202MR, T213AT } \\
\text { (1-213 aa) }\end{array}$ & $\begin{array}{c}\text { K6R, S17AT, L34V, } \\
\text { L37FL, Y43FY, Q54HQ, } \\
\text { T56IT, I63FL, K78R, } \\
\text { T79A, T83MT, N105NS, } \\
\text { R108KR, V164AT, V174T, } \\
\text { L183V, A197AT } \\
\text { (1-205aa) }\end{array}$ & $\begin{array}{c}\text { A15S, M57L, Q90K, } \\
\text { N117R, R120N, } \\
\text { Q127L, T130N, F162Y, } \\
\text { G198K, N206NS, } \\
\text { C213S, R254K, T377S, } \\
\text { V405I, 4464E, V499T, } \\
\text { A513S, R531K, S549G } \\
\text { (1-565a aa) }\end{array}$ \\
\hline & Pt7 & Chronic & 577000 & CC & None & None & Y93H & Y93H & $99.65 \%$ & $\begin{array}{l}\text { S7A, V48I, V51A, } \\
\text { S61A, A66G, T72I, } \\
\text { P86Q, K87A, S122G, } \\
\text { F147S, V170I } \\
\text { (1-181aa) }\end{array}$ & $\begin{array}{l}\text { K6R, S17T, L34V, } \\
\text { L37F, K44R, G49EG, } \\
\text { Q54H, K78R, H85N, } \\
\text { V138I, V164A, v174T, } \\
\text { Q176L, L183V } \\
\text { (1-194 aa) }\end{array}$ & $\begin{array}{c}\text { K6R, S17T, L34V, F36FL, } \\
\text { L37F, K44R, G49EG, } \\
\text { Q54H, T56IT, T64AT, } \\
\text { V75AV, K78R, H85NS, } \\
\text { V124GV, F127FS, V138I, } \\
\text { K139KR, V164A, V174T, } \\
\text { Q176L, Q181HQ, L183V } \\
\text { (1-205aa) }\end{array}$ & $\begin{array}{c}\text { A15S, M57L, Q90K, } \\
\text { V116, N117, R120N, } \\
\text { Q127L, T130N, } \\
\text { V147IV, F162Y, } \\
\text { S189PS, G198KR, } \\
\text { C213S, R254K, T377S, } \\
\text { V405IV, A421V, I424V, } \\
\text { T427P, Q464E, V499T, } \\
\text { A513S, , T520MT, } \\
\text { Q544R, S549G, L564V, } \\
\text { S565P } \\
\text { (1-569 aa) }\end{array}$ \\
\hline & Pt9 & Chronic & 94600 & CC & None & None & None & None & None & $\begin{array}{c}\text { S7A, L14F, V48I, } \\
\text { V51A, S61A, A66G, } \\
\text { P86Q, K87A, F147S, } \\
\text { S174A } \\
(1-180 \mathrm{aa})\end{array}$ & $\begin{array}{l}\text { K6R, S17T, L34V, } \\
\text { L37F, Q54H, V75A, } \\
\text { K78R, T83M, Y161H, } \\
\text { V164A, V174T } \\
\text { (1-185aa) }\end{array}$ & $\begin{array}{c}\text { K6R, I12IL, S17T, K26KR, } \\
\text { L34V, P35LP, L37F, } \\
\text { Q54H, V75A, K78R, } \\
\text { C80CR, T83M, } \\
\text { H85HCRY, A92AS, } \\
\text { T99AT, P102LP, R108KR, } \\
\text { Y161H, V164A, V174T, } \\
\text { A197AT (1-205aa) }\end{array}$ & $\begin{array}{c}\text { A15S, M57L, Q90K, } \\
\text { N110S, V116, N117R, } \\
\text { R120N, Q127L, F162Y, } \\
\text { K270R, T312S, L314S, } \\
\text { V315A, A333AV, } \\
\text { S335N, T377S, V405I, } \\
\text { K441Q, Q464E, } \\
\text { V499T, A513S, K535R, } \\
\text { S549G (1-568aa) }\end{array}$ \\
\hline \multirow[t]{2}{*}{ TC4 } & Pt8 & Chronic & 219000 & СТ & None & None & $\begin{array}{l}\text { R30Q } \\
\text { L31M } \\
\text { Y93H }\end{array}$ & $\begin{array}{l}\text { R30Q } \\
\text { L31M } \\
\text { Y93H }\end{array}$ & $\begin{array}{l}98.8 \% \\
98.8 \% \\
99.1 \%\end{array}$ & $\begin{array}{c}\text { V48I, A66G, P86Q, } \\
\text { K87A, F147S, A150V, } \\
\text { I153V } \\
(1-180 \mathrm{aa})\end{array}$ & $\begin{array}{c}\text { K6R, S17T, L34V, } \\
\text { L37F, Q54H, K78R, } \\
\text { R123Q, V124I, M133V, } \\
\text { V164A, E171Q, } \\
\text { V174T, Q176L, } \\
\text { T204TP } \\
\text { (1-210aa) }\end{array}$ & $\begin{array}{c}\text { K6R, S17T, L34V, L37F, } \\
\text { Q54H, N69NT, K78R, } \\
\text { T95MT, R108KR, R123Q, } \\
\text { V124I, M133MV, } \\
\text { K139KR, V164A, E171Q, } \\
\text { V174T, Q176L, A197T, } \\
\text { L199V (1-205aa) }\end{array}$ & $\begin{array}{c}\text { A39S, M57L, R65Q, } \\
\text { Q90K, K106KR, } \\
\text { S113G, Q127L, } \\
\text { E131N, I134L, F162Y, } \\
\text { S231N, I262V, T377S, } \\
\text { A513S, R531K } \\
\text { (1-568aa) }\end{array}$ \\
\hline & Pt10 & Acute & 163 & CC & None & None & $\begin{array}{l}\text { R30Q } \\
\text { L31M } \\
\text { Y } 93 \mathrm{H} .\end{array}$ & $\begin{array}{l}\text { R30Q } \\
\text { L31M } \\
\text { Y93H }\end{array}$ & $\begin{array}{l}99.4 \% \\
99.4 \% \\
97.6 \%\end{array}$ & $\begin{array}{c}\text { V48I, A66G, P86Q, } \\
\text { K87A, F147S, A150V, } \\
\text { I153V } \\
(16-180 \text { aa) }\end{array}$ & $\begin{array}{l}\text { K6R, S17T, L34V, } \\
\text { L37F, Q54H, K78R, } \\
\text { R123Q, V124I, M133V, } \\
\text { V164A, E171Q, } \\
\text { V174T, Q176LQ } \\
\text { (1-177aa) }\end{array}$ & $\begin{array}{l}\text { K6R, S17T, L23LP, L34V, } \\
\text { L37F, Q54H, K78R, } \\
\text { R123Q, V124I, M133V, } \\
\text { G155EG, V164A, E171Q, } \\
\text { V174T, Q176L, A197T, } \\
\text { L199V (1-205aa) }\end{array}$ & $\begin{array}{l}\text { F162Y, S231N, I262V, } \\
\text { T377S, T403AT, } \\
\text { A513S, R531K } \\
\text { (153-550aa) }\end{array}$ \\
\hline
\end{tabular}


Table 2. Cont.

\begin{tabular}{|c|c|c|c|c|c|c|c|c|c|c|c|c|c|}
\hline \multirow{2}{*}{$\begin{array}{l}\text { Cluster } \\
\text { HCV }\end{array}$} & \multirow[b]{2}{*}{$\mathrm{Pt}$} & \multirow{2}{*}{$\begin{array}{c}\mathrm{HCV} \\
\text { Infection }\end{array}$} & \multirow{2}{*}{$\begin{array}{l}\text { HCV-RNA } \\
\text { (IU/mL) }\end{array}$} & \multirow[b]{2}{*}{ IL 28} & \multicolumn{5}{|c|}{ RAS } & \multicolumn{4}{|c|}{ Other Mutations } \\
\hline & & & & & $\begin{array}{c}\text { NS3 } \\
\text { Sanger }\end{array}$ & $\begin{array}{l}\text { NS5B } \\
\text { Sanger }\end{array}$ & $\begin{array}{l}\text { NS5A } \\
\text { Sanger }\end{array}$ & $\begin{array}{l}\text { NS5A } \\
\text { NGS }\end{array}$ & $\begin{array}{c}\% \\
\text { NGS }\end{array}$ & $\begin{array}{c}\text { NS3 } \\
\text { Sanger }\end{array}$ & $\begin{array}{l}\text { NS5A } \\
\text { Sanger }\end{array}$ & $\begin{array}{c}\text { NS5A } \\
\text { NGS 1\% }\end{array}$ & $\begin{array}{l}\text { NS5B } \\
\text { Sanger }\end{array}$ \\
\hline \multirow{4}{*}{ TC5 } & Pt11 & Acute & 334000 & CC & None & None & None & None & None & $\begin{array}{l}\text { S7A, L14F, S61A, } \\
\text { T72TI, D103ND, } \\
\text { R118RW, S122T } \\
\text { (181aa) }\end{array}$ & $\begin{array}{l}\text { K6R, S17T, L34V, } \\
\text { L37F, T83M, V138I, } \\
\text { V164A, V114T, A197T } \\
\text { (1-213aa) }\end{array}$ & $\begin{array}{l}\text { K6R, S17T, L34V, L37F, } \\
\text { V46IV, V75FV, T83M, } \\
\text { H85HFLY, V138I, V164A, } \\
\text { V174T, A197T } \\
\text { (1-205aa) }\end{array}$ & $\begin{array}{c}\text { T19S, L31IV, L36M, } \\
\text { L47Q, N117R, R120N, } \\
\text { T130N, T132S, F162Y, } \\
\text { G198K, E202D, } \\
\text { A207T, A210S, A218S, } \\
\text { N231S, A300S, V321I } \\
\text { E3333, K355Q, } \\
\text { Q464E, V499T, R510K, } \\
\text { S549G } \\
\text { (1-570aa) }\end{array}$ \\
\hline & Pt12 & Acute & 16100 & $\mathrm{CC}$ & None & None & Y93HY & Y93H & $99.2 \%$ & $\begin{array}{c}\text { S7A, L14F, S61A, } \\
\text { S122T } \\
\text { (181aa) }\end{array}$ & $\begin{array}{l}\text { K6R, S17T, L34V, } \\
\text { L37F, T55AT, T83M, } \\
\text { P89LP, H128HY, } \\
\text { V138I, V164A, V174T, } \\
\text { A197T (1-206aa) }\end{array}$ & $\begin{array}{c}\text { K6R, S17AT, L34V, L37F, } \\
\text { M53MV, T83M, V138I, } \\
\text { V164A, V174T, A197T } \\
\text { (1-205aa) }\end{array}$ & $\begin{array}{c}\text { T19S, L36M, L47Q, } \\
\text { N117R, R120N, } \\
\text { T130N, T132S, G198K, } \\
\text { E202D, A207T, A210S, } \\
\text { A218S, N231S, A300T, , N } \\
\text { V321I, E333A, K355, } \\
\text { E437KE, Q464E, } \\
\text { V499T, R510K, Q514R, } \\
\text { S549G } \\
\text { (1-580aa) }\end{array}$ \\
\hline & Pt13 & Acute & 19690 & СT & None & None & None & None & None & $\begin{array}{c}\text { S7A, V48I, S61A, } \\
\text { S122T } \\
(1-140 \mathrm{aa})\end{array}$ & $\begin{array}{l}\text { K6R, S17T, L34V, } \\
\text { L37F, T56IT, T83M, } \\
\text { A92T, T135A, V138I, } \\
\text { V164A, V174T } \\
\text { (1-186aa) }\end{array}$ & $\begin{array}{c}\text { K6R, S17T, L34V, L37F, } \\
\text { T83M, A92T, T135A, } \\
\text { V138I, V164A, V174T, } \\
\text { A197AT, T200AT } \\
\text { (1-205aa) }\end{array}$ & $\begin{array}{c}\text { A16TA, T19S, L36M, } \\
\text { L47Q, R98K, N117R, } \\
\text { R120N, , T130N, } \\
\text { E131EG, T132S, } \\
\text { F162Y, G198K, E202D, } \\
\text { A207T, A210S, A218S, } \\
\text { N231S, C242S, A300S, } \\
\text { V321I, E333A, K355, } \\
\text { K4411, Q464E, } \\
\text { V499T, R510K, S549G } \\
\text { (1-570aa) }\end{array}$ \\
\hline & Pt15 & Acute & 1620000 & NA & None & None & None & None & None & $\begin{array}{l}\text { S7A, L13LF, L14F, } \\
\text { S42SF, S61A, S122T, } \\
\text { S93SF, S133SF } \\
\text { (181aa) }\end{array}$ & $\begin{array}{l}\text { K6R, S17T, L34V, } \\
\text { L37F, T83M, V138I, } \\
\text { V174T, A197T } \\
\text { (1-213aa) }\end{array}$ & $\begin{array}{c}\text { K6R, S17T, L34V, L37F, } \\
\text { Q54HQ, T83M, A92AT, } \\
\text { N105NS, V124GV, } \\
\text { T135AT, V138I, R157LR, } \\
\text { V164AV, V174T, P192PS, } \\
\text { A197T, D205DE } \\
\text { (1-205aa) }\end{array}$ & $\begin{array}{c}\text { A16T, T19S, L36M, } \\
\text { L47Q, R98K, N117R, } \\
\text { R120N, T130N, T132S, } \\
\text { F162Y, T181N, G189K, } \\
\text { E202D, A207T, A210S, } \\
\text { A218S, N231S, A300S, } \\
\text { V321I, G328EG, } \\
\text { E333A, K335Q, } \\
\text { K441Q, Q464E, } \\
\text { V499T, R510K, S554G, } \\
\text { W574L, L588S } \\
\text { (1-589aa) }\end{array}$ \\
\hline
\end{tabular}

Abbreviations: TC, transmission cluster; Pt, patient; RAS, resistance associated substitution; NGS, next-generation-sequencing. Genotype $1 \mathrm{~b}$ sequences were used as a reference. NS5A ultra-deep consensus sequences were reported as $1 \%$ cutoff. 


\subsection{Common Patterns of Variants Transmitted within the Same Cluster}

Furthermore, in order to better characterize the variants transmission, we analysed common patterns of variants shared between patients within the same cluster. For each cluster, common patterns of variants were identified (see Table S1). The number of mutations and common patterns differed according to the different cutoff of detection of minority variants used and whether the cluster was composed only of patients with acute $\mathrm{HCV}$ infection, acute and chronic $\mathrm{HCV}$ infection, or only chronic $\mathrm{HCV}$ infection. A higher number of mutations was observed in chronic $\mathrm{HCV}$ infected patients at $1 \%$ cutoff (a median of 20.5 mutations vs a median of 13 mutations in acute patients). A higher number of common patterns was observed in clusters made up of patients with only acute or acute and chronic $\mathrm{HCV}$ infection at a $15 \%$ cutoff (> $>81.82 \%$ vs. 56.21 in the cluster of only chronic HCV infected patients). Interestingly, cluster 4, containing one acute patient and the only chronic patient who had previously failed a DAA regimen, was the cluster with the highest percentage of sequence similarity within the common patterns $(81.82 \%$ at $1 \%$ cutoff and $100 \%$ at $15 \%$ cutoff) whereas cluster 3 , composed of only chronic patients, was the cluster with lowest percentage of sequence similarity (39.11\% at $1 \%$ cutoff and $56.21 \%$ at $15 \%$ ) (Table 3 ).

Table 3. Common patterns transmitted within the same clusters according to different cutoff of minority variants

\begin{tabular}{|c|c|c|c|c|c|c|c|c|}
\hline \multirow{2}{*}{$\begin{array}{c}\text { Cluster } \\
\text { HCV }\end{array}$} & \multirow{2}{*}{$\mathbf{P t}$} & \multirow{2}{*}{$\begin{array}{c}\mathrm{HCV} \\
\text { Infection }\end{array}$} & \multicolumn{2}{|c|}{ Cut-off 1\% } & \multicolumn{2}{|c|}{ Cut-off $5 \%$} & \multicolumn{2}{|c|}{ Cut-off 15\% } \\
\hline & & & $\underset{\text { Mutations }}{\mathrm{N}}$ & $\begin{array}{l}\text { Common } \\
\text { Patterns \% }\end{array}$ & $\begin{array}{c}\mathbf{N} \\
\text { Mutations }\end{array}$ & $\begin{array}{l}\text { Common } \\
\text { Patterns \% }\end{array}$ & $\underset{\text { Mutations }}{\mathrm{N}}$ & $\begin{array}{c}\text { Common } \\
\text { Patterns \% }\end{array}$ \\
\hline \multirow{2}{*}{ TC1 } & Pt1 & Chronic & 20 & \multirow[b]{2}{*}{50.00} & 13 & \multirow{2}{*}{69.23} & 11 & \multirow{2}{*}{81.82} \\
\hline & Pt2 & Acute & 10 & & 10 & & 10 & \\
\hline \multirow{3}{*}{ TC2 } & Pt3 & Chronic & 20 & \multirow{3}{*}{70.00} & 16 & \multirow{3}{*}{81.25} & 16 & \multirow{3}{*}{81.25} \\
\hline & Pt4 & Acute & 14 & & 13 & & 13 & \\
\hline & Pt5 & Acute & 20 & & 14 & & 14 & \\
\hline \multirow{2}{*}{ TC4 } & Pt8 & Chronic & 22 & \multirow{2}{*}{81.82} & 20 & \multirow{2}{*}{90.00} & 18 & \multirow{2}{*}{100} \\
\hline & Pt10 & Acute & 20 & & 18 & & 18 & \\
\hline \multirow{3}{*}{ TC3 } & Pt6 & Chronic & 17 & \multirow{3}{*}{39.11} & 13 & \multirow{3}{*}{55.87} & 11 & \multirow{3}{*}{56.21} \\
\hline & Pt7 & Chronic & 23 & & 15 & & 15 & \\
\hline & Pt9 & Chronic & 21 & & 14 & & 12 & \\
\hline \multirow{4}{*}{ TC5 } & Pt11 & Acute & 12 & \multirow{4}{*}{70.33} & 10 & \multirow{4}{*}{80.72} & 10 & \multirow{4}{*}{85.91} \\
\hline & Pt12 & Acute & 11 & & 11 & & 11 & \\
\hline & Pt13 & Acute & 12 & & 11 & & 11 & \\
\hline & Pt15 & Acute & 17 & & 13 & & 10 & \\
\hline
\end{tabular}

Abbreviations: TC, transmission cluster; $\mathrm{Pt}$, patient. Common patterns within the same clusters have been analyzed at different cutoff of detection of minority variants, between acute vs chronic (clusters 1, 2, 4); or between chronic infected patients (cluster 3), or between acute infected patients (cluster 5).

\subsection{Nucleotide Sequence Variability and Shannon Entropy}

Generally, the intra-host HCV nucleotide sequence variability (NSV) obtained by using all NGS prevalence cutoffs $(1 \%, 2 \%, 5 \%, 10 \%, 15 \%)$ was always higher in patients with $\mathrm{HCV}$ chronic infection vs patients with HCV acute infection $(p<0.05$ ), with the highest difference at $1 \%$ cutoff (median [IQR] NSV 14.1 [13.3-14.8] in patients with chronic HCV infection vs 1.3 [0.8-2.9] in patients with acute HCV infection, $p=0.0016$ ) (Figure 2).

The Shannon entropy showed a similar trend, with generally higher values in patients with chronic $\mathrm{HCV}$ infection vs patients with acute $\mathrm{HCV}$ infection, however, significantly higher values were observed only when comparing $\beta$-thalassemia patients with chronic infection versus $\beta$-thalassemia patients with acute HCV infection (Sn median HCV chronic 0.66 [IQR 0.60-0.81] vs Sn median HCV acute 0.34 [IQR 0.27-0.36] $p=0.01$ ). In contrast, no significant difference was observed between $S n$ values from $\mathrm{HCV}$ acute onco-hematologic-patients vs HCV chronic $\beta$-thalassemia-patients (Sn median 0.55 [IQR 0.54-0.60] and Sn median 0.66 [IQR 0.60-0.81], respectively). 

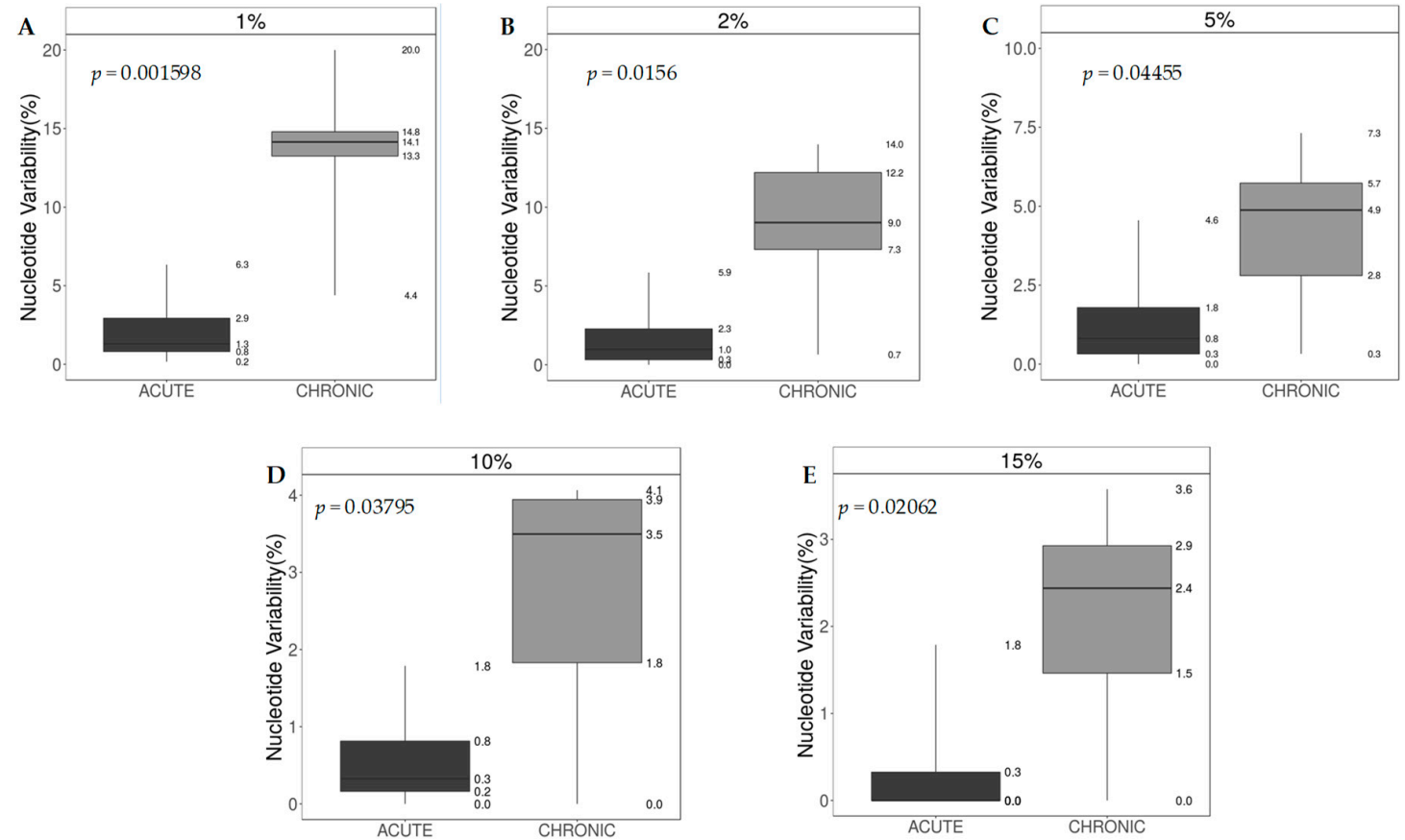

Figure 2. NS5A nucleotide sequence variability (\%), Acute vs Chronic. Difference between acute and chronic infected patients in Nucleotide-sequence-variability at different NGS cutoff of detection of minority variants. The $p$-value was calculated using the Mann-Whitney U Test. (A) nucleotide sequence variability at $1 \%$; (B) nucleotide sequence variability at $2 \%$; (C) nucleotide sequence variability at $5 \%$; (D) nucleotide sequence variability at 10\%; Panel E: nucleotide sequence variability at $15 \%$.

Interestingly, different Shannon Entropy median values were also observed between HCV sequences of acute onco-hematologic-patients vs HCV sequences of acute $\beta$-thalassemia-patients (Sn median 0.55 [IQR 0.54-0.60] vs median 0.34 [IQR 0.27-0.36] $p=0.02$ ) (Table 4), indicating a higher HCV sequence heterogeneity in patients with HCV acute infection in the presence of an onco-hematology disease in comparison to patients with HCV acute infection in the presence of $\beta$-thalassemia. This observation was also confirmed as a significant trend by analysing the intra-host NSV between the two groups of HCV acute patients with the two different diseases (see Figure S1).

Table 4. Amino Acid Variability was estimated for each NS5A amino acid position for each patient.

\begin{tabular}{|c|c|c|c|c|c|c|}
\hline Patient ID & $\begin{array}{c}\mathrm{HCV} \\
\text { Infection }\end{array}$ & $\begin{array}{l}\text { Number } \\
\text { Cluster }\end{array}$ & Sn ${ }^{a}$ & $\begin{array}{l}\text { Overall HCV } \\
\text { Infection }\end{array}$ & Sn Median (IQR) & $p$ Value $^{\mathrm{b}}$ \\
\hline \multicolumn{7}{|c|}{ Patients with $\beta$-thalassemia } \\
\hline 1 & Acute & 1 & 0.23 & & & \\
\hline 4 & Acute & 2 & 0.35 & & & \\
\hline 5 & Acute & 2 & 0.36 & Acute & $0.34(0.27-0.36)$ & \\
\hline 10 & Acute & 4 & 0.31 & & & \\
\hline 2 & Chronic & 1 & 0.65 & & & $0.01^{\mathrm{c}}$ \\
\hline 3 & Chronic & 2 & 0.93 & & & \\
\hline 6 & Chronic & 3 & 0.66 & & & \\
\hline 7 & Chronic & 3 & 0.80 & Chronic & $0.66(0.60-0.81)$ & \\
\hline 9 & Chronic & 3 & 0.59 & & & \\
\hline 8 & Chronic & 4 & 0.38 & & & \\
\hline \multicolumn{7}{|c|}{ Patients with onco-hematologic disease } \\
\hline 11 & Acute & 5 & 0.59 & & & \\
\hline 12 & Acute & 5 & 0.54 & & & \\
\hline 13 & Acute & 5 & 0.41 & Acute & $0.55(0.54-0.60)$ & $0.02^{\mathrm{d}}$ \\
\hline 14 & Acute & Out of cluster & 0.68 & & & \\
\hline 15 & Acute & 5 & 0.53 & & & \\
\hline
\end{tabular}

${ }^{a}$ Sn, Shannon Entropy weighted for the intra-patient prevalence of viral species, ${ }^{b} p$ value was calculated using the Mann-Whitney test. ${ }^{c}$ Acute vs chronic; ${ }^{\mathrm{d}}$ acute vs acute. 


\subsection{IL28B Polymorphisms and Intra-Host HCV Variability}

To better characterize a potential virus-host interaction, we also investigated the relationship between human polymorphisms IL-28B rs12979860 and the presence of $Y 93 \mathrm{H}$, the nucleotide sequence variability (NSV) and the Shannon entropy. Among the 15 patients analysed, the IL-28B information was available for 14 patients (13 of whom were involved in the clusters, see Table 2). Overall, CC polymorphism was present in seven patients and CT polymorphism in the remaining seven patients. No specific relationship between IL-28 (CC and CT) and Y93H, NSV and Shannon Entropy were identified with any statistically significant difference ( $p>0.05$; data not shown).

\section{Discussion}

The use of molecular methodologies based on viral gene sequencing and phylogenetic analysis is the best approach to identify and confirm potential suspected viral transmission events, by assessing genetic viral similarities within the individuals allegedly involved. A number of studies have shown that a phylogenetic tree analysis of individual HCV RNA isolates provides clear documentation of HCV transmissions occurring within healthcare settings. In 2006, molecular phylogenetic analysis was used to prove the innocence of foreign medical staff accused of transmitting HIV strains to children being treated in a hospital in Benghazi, in Libya [30]. In that study, in fact, researchers showed that both HCV and HIV-1 strains were already circulating in the hospital, before the arrival of the foreign medical staff in March 1998, suggesting that the hospital had a long-standing nosocomial infection control problem [30]. Several other nosocomial HCV transmission scenarios have been observed, with HCV infection transmission between haemodialysis patients [51-56], or among hematologic patients, generally through inappropriate use of medical devices (e.g., the inappropriate reuse of saline flush syringes) [57] and in patients with $\beta$-thalassemia [58,59]. However, the number of reported transmissions during the acute phase is limited, mainly because acute HCV infections are not always detected, due to the asymptomatic nature of the infection. Recently, an outbreak of HCV transmission in a Dutch haemodialysis unit was documented through the use of NS5A gene sequencing [10]. We have also recently identified separate episodes of $\mathrm{HCV}$ transmission in two healthcare settings by performing a phylogenetic analysis on NS3, NS5A and NS5B genes using Sanger sequencing: one case regarding patients with acute HCV infection diagnosis affected by onco-hematologic disease [36] and the second case in patients with acute HCV infection diagnosis with $\beta$-thalassemia [31]. In this present study, through NS5A NGS analysis we aimed to investigate the intra-host viral quasispecies composition in 15 patients, all of whom were putatively involved in transmission clusters. The Bayesian analysis on NS5A gene confirmed the five transmission clusters considered epidemiologically monophyletic clades, by a posterior probability $\geq 90$. Among the 15 patients analyzed, 14 patients were involved in transmission clusters, composed of between two and four patients per cluster. Among the five onco-hematologic patients, all of whom showing acute HCV infection in the same hospital during the same period, the NGS analysis confirmed a transmission cluster involving only four patients, with one patient outside the cluster, for whom no evidence of minority HCV variants in common with the other four patients was found. The other transmission clusters concerned $\beta$-thalassemia patients with acute and/or chronic HCV state of infection. Notably, three transmission clusters involved both acute and chronic HCV infected patients and one cluster involved three HCV chronic patients, all of whom were being treated in the same clinical center, during the same period. NGS analysis confirmed that all individuals were infected with HCV GT1b subtype, without cases of minority variants of other genotypes and/or HCV subtypes, excluding cases of coinfection. Overall, the Bayesian phylogenetic tree performed on NGS NS5A sequences confirmed the previous results, suggesting that the viruses present in the identified clusters have such a high degree of similarity that they can be assumed to have a common origin.

Specific NS5A RASs (R30Q, L31M and/or Y93H) were previously observed using Sanger sequencing in all five transmission clusters. Through NGS, the patterns of resistance were confirmed, and no additional minority NS5A-resistant variants were identified at any of the cutoffs used (up to $1 \%$ ). In all 
clusters at least one patient showed the $\mathrm{Y} 93 \mathrm{H}$, even if it was not shared within all patients involved in the same transmission cluster. This RAS was present overall in $37.5 \%$ of patients with acute $\mathrm{HCV}$ infection and in $66.7 \%$ of patients with chronic HCV infection. This prevalence is quite high, in all probability due mainly to these existing nosocomial transmission events. In fact, this level of prevalence is higher than the natural prevalence recently reported in a large Italian cohort of DAA naïve patients infected with HCV GT1b, which was around 10\% [23]. In one cluster (TC2) the patient with chronic $\mathrm{HCV}$ infection showed the $\mathrm{Y} 93 \mathrm{H}$ at $32.9 \%$ of intra-patient prevalence, and this mutation was not present in the other two patients with acute HCV infection, who were in the same transmission cluster. In this case, it can be presumed that the wild-type variant from the individual with the chronic infection (around 70\% of intra-patient prevalence) was the major variant transmitted in the two individuals who acquired the acute HCV infection. In contrast, the patients with acute HCV infection found in transmission clusters TC1 and TC4 showed the same RAVs, with the same high intra-patient prevalence rate, as observed in the patients with chronic HCV infection belonging to the same clusters (>97.0\%). In both cases, there is virological evidence of resistance transmission. For instance, in TC4, the patient with a chronic infection (Pt8) had previously failed a DAA treatment containing a NS5A inhibitor (grazoprevir/elbasvir) and developed at failure a resistant HCV with three NS5A RASs (R30Q, L31M and $\mathrm{Y} 93 \mathrm{H})$, which was fully transmitted to the patient who acquired the acute HCV infection (Pt10). Even if this case is, to our knowledge, the first case of nosocomial transmission of an HCV resistant variant, it is important to mention that a case of the transmission of an HCV NS3 resistant variant from a telaprevir treated patient to his sexual HIV positive partner has already been observed. [60].

To uncover the potential mechanisms of the intra-host variability observed within the nosocomial transmission clusters identified, we investigated the relationship between $\mathrm{Y} 93 \mathrm{H}$ and the IL-28 polymorphisms in the rs12979860 gene. A previous study analysing the prevalence of resistant variants in a large European population of HCV infected individuals, in correlation to SNPs in IFNL4, in fact showed that the NS5A resistant variant Y93H was detected frequently in HCV GT $1 \mathrm{~b}(14 \%)$ and it was significantly associated with the beneficial rs12979860 IL-28B polymorphism CC [61]. In our study, however, probably due to the limited numbers of cases analysed, even though all the individuals were infected with HCV GT 1b, we did not find any specific and statistically significant association between $\mathrm{Y} 93 \mathrm{H}$ and the IL-28B polymorphism CC.

To better characterize the virus host interactions, we also analysed the HCV variability and Shannon entropy in individuals with acute HCV infection in comparison to individuals with chronic infection. As expected, the NSV was significantly higher in patients with chronic HCV infection versus patients with acute infection, at all cutoffs tested $(p<0.05)$, with the highest difference at $1 \%$ cutoff, indicating that HCV infection over a longer period of time led to higher levels of viral evolution and quasispecies diversity.

Analyzing both groups (acute vs chronic infection), with respect also to the underlying disease ( $\beta$-thalassemia vs onco-hematologic disease), the HCV sequence heterogeneity, measured as Shannon Entropy, was significantly higher in chronic HCV patients in comparison to patients with acute infection, but only in the presence of $\beta$-thalassemia $(p=0.01)$. Significantly higher Shannon entropy was also observed analysing the sequences of HCV acute patients with onco-hematologic disease respect to $\mathrm{HCV}$ acute patients with $\beta$-thalassemia. Instead, similar levels of sequence heterogeneity were observed between $\mathrm{HCV}$ acute patients with onco-hematologic disease and chronic HCV patients with $\beta$-thalassemia.

This observation can be explained by the compromised immune system of patients with onco-hematologic disease, who did not in fact develop anti HCV antibodies after the infection (also at the last follow-up visits). These patients therefore fought the spread of the virus less efficiently, thus allowing it to change more easily with high replication turn-over, similar to that which would occur in a patient with a chronic infection. For instance, the HCV-RNA levels corresponding to the samples of HCV sequencing of the acute patients with onco-hematologic disease were higher than the levels observed in the acute HCV infected patients with $\beta$-thalassemia. On the other hand, 
the patients with $\beta$-thalassemia during the acute infection were under strong immune pressure, effectively limiting the viral replication over such a short period, and as a consequence also limiting variability and heterogeneity.

It is worth mentioning that the only $\beta$-thalassemia patient with $\mathrm{HCV}$ chronic infection that previously failed a DAA-treatment $(\mathrm{Pt} 8)$ had a low value of Shannon Entropy, at similar levels to that observed in the other acute patients with $\beta$-thalassemia ( $\mathrm{Sn}$ in Pt8 0.38 versus Sn median in HCV acute $\beta$-thalassemia patients 0.34 [IQR $0.27-0.36]$ ) (Table 3), and this could be the consequence of the strong pharmacological pressure that allowed the selection and transmission of the resistant variants to the patient with acute HCV infection belonging to the same transmission cluster (TC4). Cluster TC4 was in fact the cluster with the highest percentage of common patterns at all of the cutoffs analysed (from $81.8 \%$ at $1 \%$ to $100 \%$ at $15 \%$ ).

In conclusion, even though this study is limited to a small number of individuals, we highlighted the presence of nosocomial transmission clusters in patients with a diagnosis of acute $\mathrm{HCV}$ infection. Transmission of treatment resistant HCV variants in some cases occurred, and the transmission/maintenance of Y93H RAS in acute infected individuals was independent of the IL-28 polymorphism. We also observed a differential degree of HCV sequence variability and heterogeneity, which was higher in patients with chronic HCV infection, followed by immunocompromised patients during acute infection, and lower in immunocompetent patients during the acute HCV infection. Further studies are needed to better understand the mechanisms driving the intra host interactions and the transmission/maintenance of resistance.

Supplementary Materials: The following are available online at http://www.mdpi.com/2073-4409/8/7/666/s1, Figure S1, Table S1, Texts S1-S2 [62,63].

Author Contributions: F.C.-S., G.B., C.F.P, A.C., G.B.G., and V.D.M conceived the analysis. M.C.B. and M.A. developed and conducted the analyses. M.C.B., L.C., L.F., S.B., performed the NGS, bioinformatics and phylogenetic analyses. V.C.D.M., contributed to the statistical analyses. M.C.S. and M.A performed the HCV Sanger-sequencing analysis. R.M.P., G.B., F.B., S.G., W.M., F.F., M.C., A.P., G.B.G and V.D.M., enrolled patients and collected clinical data. M.C.B., M.A., L.C. and F.C.-S. wrote the manuscript. All authors reviewed and approved the manuscript.

Funding: This work was supported by the Italian Ministry of Instruction, University and Research (MIUR) (Bandiera InterOmics Protocollo PB05 $1^{\circ}$ ), by the Italian Ministry of Health: RF-2016-02362422 and by Aviralia and Vironet $C$ Foundation. The funders had no role in study design, data collection and analysis, decision to publish, or preparation of the manuscript.

Acknowledgments: We gratefully thank the Molecular Virology group of the Tor Vergata University, Katia Yu La Rosa, Riccardo Campoli, Rossana Scutari and Lorenzo Piermatteo for technical support.

Conflicts of Interest: The authors declare no conflict of interest.

\section{References}

1. WHO World Health Organization Hepatitis C Virus Factsheet. Available online: https://www.who.int/en/ news-room/fact-sheets/detail/hepatitis-c (accessed on 1 February 2019).

2. Centers for Disease Control and Prevention (CDC); National Notifiable Diseases Surveillance System (NNDSS). Hepatitis C, Acute: 2016 Case Definition. Available online: https://wwwn.cdc.gov/nndss/ conditions/hepatitis-c-acute/case-definition/2016/ (accessed on 2 April 2019).

3. Fox, R.K.; Spach, D.H. Diagnosis of Acute HCV Infection. Available online: https://www.hepatitisc.uw.edu/ go/screening-diagnosis/acute-diagnosis/core-concept/all (accessed on 2 April 2019).

4. Pawlotsky, J.-M.; Negro, F.; Aghemo, A.; Berenguer, M.; Dalgard, O.; Dusheiko, G.; Marra, F.; Puoti, M.; Wedemeyer, H. EASL Recommendations on Treatment of Hepatitis C. J. Hepatol. 2018, 69, 461-511. [CrossRef] [PubMed]

5. Thein, H.-H.; Yi, Q.; Dore, G.J.; Krahn, M.D. Estimation of stage-specific fibrosis progression rates in chronic hepatitis C virus infection: A meta-analysis and meta-regression. Hepatology 2008, 48, 418-431. [CrossRef] [PubMed]

6. Friedman, S.L. Evolving challenges in hepatic fibrosis. Nat. Rev. Gastroenterol. Hepatol. 2010, 7, 425-436. [CrossRef] [PubMed] 
7. Pozzetto, B.; Memmi, M.; Garraud, O.; Roblin, X.; Berthelot, P.; Pozzetto, B.; Memmi, M.; Garraud, O.; Berthelot, P.; Immunité, G. Health care-associated hepatitis C virus infection. World J. Gastroenterol. 2014, 20, 17265-17278. [CrossRef] [PubMed]

8. Prati, D. Transmission of hepatitis $C$ virus by blood transfusions and other medical procedures: A global review. J. Hepatol. 2006, 45, 607-616. [CrossRef] [PubMed]

9. Johannessen, I.; Danial, J.; Smith, D.B.; Richards, J.; Imrie, L.; Rankin, A.; Willocks, L.J.; Evans, C.; Leen, C.; Gibson, P.; et al. Molecular and epidemiological evidence of patient-to-patient hepatitis $\mathrm{C}$ virus transmission in a Scottish emergency department. J. Hosp. Infect. 2018, 98, 412-418. [CrossRef] [PubMed]

10. Heikens, E.; Hetem, D.J.; Jousma-rutjes, J.P.W.; Nijhuis, W.; Boland, G.J.; Hommes, N.H.; Thang, O.H.D.; Schuurman, R. Hepatitis C virus transmission in a Dutch haemodialysis unit: Detailed outbreak investigation using NS5A gene sequencing. J. Hosp. Infect. 2019, 101, 333-338. [CrossRef]

11. Smith, D.B.; Bukh, J.; Kuiken, C.; Muerhoff, A.S.; Rice, C.M.; Stapleton, J.T.; Simmonds, P. Expanded classification of hepatitis $C$ virus into 7 genotypes and 67 subtypes: Updated criteria and genotype assignment web resource. Hepatology 2014, 59, 318-327. [CrossRef]

12. Borgia, S.M.; Hedskog, C.; Parhy, B.; Hyland, R.H.; Stamm, L.M.; Brainard, D.M.; Subramanian, M.G.; McHutchison, J.G.; Mo, H.; Svarovskaia, E.; et al. Identification of a Novel Hepatitis C Virus Genotype From Punjab, India: Expanding Classification of Hepatitis C Virus Into 8 Genotypes. J. Infect. Dis. 2018, 218, 1722-1729. [CrossRef]

13. Hedskog, C.; Parhy, B.; Chang, S.; Zeuzem, S.; Moreno, C.; Shafran, S.D.; Borgia, S.M.; Asselah, T.; Alric, L.; Abergel, A.; et al. Identification of 19 Novel Hepatitis C Virus Subtypes-Further Expanding HCV Classification. Open Forum Infect. Dis. 2019, 6, ofz076. [CrossRef]

14. Messina, J.P.; Humphreys, I.; Flaxman, A.; Brown, A.; Cooke, G.S.; Pybus, O.G.; Barnes, E. Global distribution and prevalence of hepatitis $C$ virus genotypes. Hepatology 2015, 61,77-87. [CrossRef]

15. Bukh, J. The history of hepatitis $\mathrm{C}$ virus (HCV): Basic research reveals unique features in phylogeny, evolution and the viral life cycle with new perspectives for epidemic control. J. Hepatol. 2016, 65, S2-S21. [CrossRef]

16. Mancusi, R.L.; Andreoni, M.; d'Angela, D.; Sarrecchia, C.; Spandonaro, F. Epidemiological burden estimates for pathologies with a nonconstant risk: An application to HCV in Italy according to age, Metavir score, and genotype: A systematic review and meta-analysis. Medicine (Baltimore) 2016, 95, e5143. [CrossRef]

17. Progetto PITER. Available online: http://www.progettopiter.it/ (accessed on 9 May 2019).

18. Rossetti, B.; Bai, F.; Tavelli, A.; Galli, M.; Antinori, A.; Castelli, F.; Pellizzer, G.; Cozzi-Lepri, A.; Bonora, S.; d'Arminio Monforte, A.; et al. Evolution of the prevalence of hepatitis $C$ virus infection and hepatitis $C$ virus genotype distribution in human immunodeficiency virus-infected patients in Italy between 1997 and 2015. Clin. Microbiol. Infect. 2018, 24, 422-427. [CrossRef]

19. Kartashev, V.; Döring, M.; Nieto, L.; Coletta, E.; Kaiser, R.; Sierra, S.; Guerrero, A.; Stoiber, H.; Paar, C.; Vandamme, A.M.; et al. New findings in HCV genotype distribution in selected West European, Russian and Israeli regions. J. Clin. Virol. 2016, 81, 82-89. [CrossRef] [PubMed]

20. Fondazione Vironet C-Fondazione Italiana per gli Studi di Resistenza ai Farmaci anti HCV. Available online: https://www.vironetc.org/ (accessed on 9 May 2019).

21. Domingo, E.; Sheldon, J.; Perales, C. Viral Quasispecies Evolution. Microbiol. Mol. Biol. Rev. 2012, 76, $159-216$. [CrossRef]

22. Spengler, U. Direct antiviral agents ( DAAs ) - A new age in the treatment of hepatitis C virus infection. Pharmacol. Ther. 2018, 183, 118-126. [CrossRef]

23. Bertoli, A.; Sorbo, M.C.; Aragri, M.; Lenci, I.; Teti, E.; Chiara, V.; Maio, D.; Gianserra, L.; Biliotti, E.; Masetti, C.; et al. Prevalence of Single and Multiple Natural NS3, NS5A and Substitutions in Hepatitis C Virus Genotypes 1-4 in Italy. Sci. Rep. 2018, 8, 8988. [CrossRef] [PubMed]

24. Yerly, S.; Kaiser, L.; Race, E.; Bru, J.-P.; Clavel, F.; Perrin, L. Transmission of antiretroviral-drug-resistant HIV-1 variants. Lancet 1999, 354, 729-733. [CrossRef]

25. Salpini, R.; Svicher, V.; Cento, V.; Gori, C.; Bertoli, A.; Scopelliti, F.; Micheli, V.; Cappiello, T.; Spanò, A.; Rizzardini, G.; et al. Characterization of drug-resistance mutations in HBV D-genotype chronically infected patients, naïve to antiviral drugs. Antivir. Res. 2011, 92, 382-385. [CrossRef] [PubMed] 
26. Prokunina-Olsson, L.; Muchmore, B.; Tang, W.; Pfeiffer, R.M.; Park, H.; Dickensheets, H.; Hergott, D.; Porter-Gill, P.; Mumy, A.; Kohaar, I.; et al. A variant upstream of IFNL3 (IL28B) creating a new interferon gene IFNL4 is associated with impaired clearance of hepatitis C virus. Nat. Genet. 2013, 45, 164-171. [CrossRef] [PubMed]

27. Ge, D.; Fellay, J.; Thompson, A.J.; Simon, J.S.; Shianna, K.V.; Urban, T.J.; Heinzen, E.L.; Qiu, P.; Bertelsen, A.H.; Muir, A.J.; et al. Genetic variation in IL28B predicts hepatitis C treatment-induced viral clearance. Nature 2009, 461, 399-401. [CrossRef] [PubMed]

28. Thomas, D.L.; Thio, C.L.; Martin, M.P.; Qi, Y.; Ge, D.; Colm, O.; Kidd, J.; Kidd, K.; Khakoo, S.I.; Alexander, G.; et al. Genetic variation in IL28B and spontaneous clearance of hepatitis C virus. Nature 2009, 461, 798-801. [CrossRef] [PubMed]

29. Suppiah, V.; Moldovan, M.; Ahlenstiel, G.; Berg, T.; Weltman, M.; Abate, M.L.; Bassendine, M.; Spengler, U.; Dore, G.J.; Powell, E.; et al. IL28B is associated with response to chronic hepatitis C interferon- $\alpha$ and ribavirin therapy. Nat. Genet. 2009, 41, 1100-1104. [CrossRef] [PubMed]

30. de Oliveira, T.; Pybus, O.G.; Rambaut, A.; Salemi, M.; Cassol, S.; Ciccozzi, M.; Rezza, G.; Gattinara, G.C.; D'Arrigo, R.; Amicosante, M.; et al. Molecular epidemiology: HIV-1 and HCV sequences from Libyan outbreak. Nature 2006, 444, 836-837. [CrossRef] [PubMed]

31. Aragri, M.; Fabeni, L. Identification of HCV Transmission Clusters in a Group of Thalassemic Patients with Diagnosis of ACUTE HCV Infection [Abstract]. Hepatology 2018, 68, 911A-912A.

32. Barzon, L.; Lavezzo, E.; Militello, V.; Toppo, S.; Palù, G. Applications of next-generation sequencing technologies to diagnostic virology. Int. J. Mol. Sci. 2011, 12, 7861-7884. [CrossRef]

33. Guinoiseau, T.; Moreau, A.; Hohnadel, G.; Ngo-Giang-Huong, N.; Brulard, C.; Vourc'h, P.; Goudeau, A.; Gaudy-Graffin, C. Deep sequencing is an appropriate tool for the selection of unique Hepatitis C virus (HCV) variants after single genomic amplification. PLOS ONE 2017, 12, e0174852. [CrossRef]

34. Mansoor, S.; Javed, A.; Ali, A.; Mansoor, A. Heterogeneous genomic locations within NS3, NS4A and NS4B identified for genotyping and subtyping of Hepatitis $C$ virus: A simple genome analysis approach. Infect. Genet. Evol. 2016, 44, 61-68. [CrossRef]

35. Andre-Garnier, E.; Besse, B.; Rodallec, A.; Ribeyrol, O.; Ferre, V.; Luco, C.; Le Guen, L.; Bourgeois, N.; Gournay, J.; Billaud, E.; et al. An NS5A single optimized method to determine genotype, subtype and resistance profiles of Hepatitis C strains. PLOS ONE 2017, 12, e0179562. [CrossRef]

36. Brancaccio, G.; Sorbo, M.C.; Frigeri, F.; Rizzo, V.; Cantone, M.; Genderini, F.; Fabeni, L.; Pinto, A.; Perno, C.F.; Ceccherini-Silberstein, F; et al. Treatment of Acute Hepatitis C With Ledipasvir and Sofosbuvir in Patients With Hematological Malignancies Allows Early Re-start of Chemotherapy. Clin. Gastroenterol. Hepatol. 2018, 16, 977-978. [CrossRef] [PubMed]

37. Di Maio, V.C.; Cento, V.; Aragri, M.; Paolucci, S.; Pollicino, T.; Coppola, N.; Bruzzone, B.; Ghisetti, V.; Zazzi, M.; Brunetto, M.; et al. Frequent NS5A and multiclass resistance in almost all HCV genotypes at DAA failures: What are the chances for second-line regimens? J. Hepatol. 2018, 68, 597-600. [CrossRef] [PubMed]

38. Sorbo, M.C.; Cento, V.; Di Maio, V.C.; Howe, A.Y.M.; Garcia, F.; Perno, C.F.; Ceccherini-Silberstein, F. Hepatitis $\mathrm{C}$ virus drug resistance associated substitutions and their clinical relevance: Update 2018. Drug Resist. Updat. 2018, 37, 17-39. [CrossRef] [PubMed]

39. Kalaghatgi, P.; Sikorski, A.M.; Knops, E.; Rupp, D.; Sierra, S.; Heger, E.; Neumann-Fraune, M.; Beggel, B.; Walker, A.; Timm, J.; et al. Geno2pheno [HCV]-A Web-based Interpretation System to Support Hepatitis C Treatment Decisions in the Era of Direct-Acting Antiviral Agents. PLoS ONE 2016, 11, e0155869. [CrossRef] [PubMed]

40. Li, H. Aligning sequence reads, clone sequences and assembly contigs with BWA-MEM. arXiv 2013, arXiv:1303.3997.

41. bamToFreq. Available online: http://github.com/matdoering/bamToFreq (accessed on 15 November 2018).

42. Döring, M.; Büch, J.; Friedrich, G.; Pironti, A.; Kalaghatgi, P.; Knops, E.; Heger, E.; Obermeier, M.; Däumer, M.; Thielen, A.; et al. geno2pheno[ngs-freq]: A genotypic interpretation system for identifying viral drug resistance using next-generation sequencing data. Nucleic Acids Res. 2018, 46, W271-W277. [CrossRef] [PubMed]

43. Töpfer, A.; Zagordi, O.; Prabhakaran, S.; Roth, V.; Halperin, E.; Beerenwinkel, N. Probabilistic inference of viral quasispecies subject to recombination. J. Comput. Biol. 2013, 20, 113-123. [CrossRef] [PubMed] 
44. Saitou, N.; Nei, M. The neighbor-joining method: A new method for reconstructing phylogenetic trees. Mol. Biol. Evol. 1987, 4, 406-425.

45. Kimura, M. A simple method for estimating evolutionary rates of base substitutions through comparative studies of nucleotide sequences. J. Mol. Evol. 1980, 16, 111-120. [CrossRef]

46. Tavarè, S. Some probabilistic and statistical problems in the analysis of DNA sequences. Lect. Math. Life Sci. 1986, 17, 57-86.

47. FigTree. Available online: http://tree.bio.ed.ac.uk/software/figtree/ (accessed on 15 November 2018).

48. Huelsenbeck, J.P.; Ronquist, F. MRBAYES: Bayesian inference of phylogenetic trees. Bioinformatics 2001, 17, 754-755. [CrossRef] [PubMed]

49. Ronquist, F.; Huelsenbeck, J.P. MrBayes 3: Bayesian phylogenetic inference under mixed models. Bioinformatics 2003, 19, 1572-1574. [CrossRef] [PubMed]

50. R Core Team. R: A Language and Environment for Statistical Computing; R Foundation for Statistical Computing: Vienna, Austria, 2018. Available online: http://www.R-project.org/ (accessed on 15 November 2018).

51. Kokubo, S.; Horii, T.; Yonekawa, O.; Ozawa, N.; Mukaide, M. A phylogenetic-tree analysis elucidating nosocomial transmission of hepatitis C virus in a haemodialysis unit. J. Viral Hepat. 2002, 9, 450-454. [CrossRef] [PubMed]

52. Abacioglu, Y.H.; Bacaksiz, F.; Bahar, I.H.; Simmonds, P. Molecular evidence of nosocomial transmission of hepatitis C virus in a haemodialysis unit. Eur. J. Clin. Microbiol. Infect. Dis. 2000, 19, 182-186. [CrossRef] [PubMed]

53. Duong, C.M.; McLaws, M.-L. An investigation of an outbreak of hepatitis C virus infections in a low-resourced hemodialysis unit in Vietnam. Am. J. Infect. Control 2016, 44, 560-566. [CrossRef]

54. Garvey, M.I.; Bradley, C.W.; Holden, K.L.; Hewins, P.; Ngui, S.; Tedder, R.; Jumaa, P.; Smit, E. Use of genome sequencing to identify hepatitis $C$ virus transmission in a renal healthcare setting. J. Hosp. Infect. 2017, 96, 157-162. [CrossRef]

55. Nguyen, D.B.; Gutowski, J.; Ghiselli, M.; Cheng, T.; Bel Hamdounia, S.; Suryaprasad, A.; Xu, F.; Moulton-Meissner, H.; Hayden, T.; Forbi, J.C.; et al. A Large Outbreak of Hepatitis C Virus Infections in a Hemodialysis Clinic. Infect. Control Hosp. Epidemiol. 2016, 37, 125-133. [CrossRef]

56. Senatore, S.; Galli, C.; Conti, A.; Faccini, M.; Cantoni, S.; Ciconali, G.; Mainardi, G.; Lamberti, A.; Dighera, R.; Radice Trolli, F.; et al. Hepatitis C virus outbreak in a haemodialysis unit: Learning from failures. J. Hosp. Infect. 2016, 3, 249-252. [CrossRef]

57. Arnold, S.; Melville, S.K.; Morehead, B.; Vaughan, G.; Moorman, A.; Crist, M.B. Notes from the Field: Hepatitis C Transmission from Inappropriate Reuse of Saline Flush Syringes for Multiple Patients in an Acute Care General Hospital-Texas, 2015. MMWR. Morb. Mortal. Wkly. Rep. 2017, 66, 258-260. [CrossRef]

58. Maria Ricerca, B.; Di Girolamo, A. Infections in Thalassemia a Therapy-Related Complications. Mediterr. J. Hematol. Infect. Dis. 2009, 1, 2009028.

59. Ahmed Kiani, R.; Anwar, M.; Waheed, U.; Asad, M.J.; Abbasi, S.; Abbas Zaheer, H. Epidemiology of Transfusion Transmitted Infection among Patients with $\beta$-Thalassaemia Major in Pakistan. J. Blood Transfus. 2016, 2016, 1-5. [CrossRef] [PubMed]

60. Franco, S.; Tural, C.; Nevot, M.; Moltó, J.; Rockstroh, J.K.; Clotet, B.; Martinez, M.A. Detection of a sexually transmitted hepatitis $C$ virus protease inhibitor-resistance variant in a human immunodeficiency virus-infected homosexual man. Gastroenterology 2014, 147, 599-601. [CrossRef] [PubMed]

61. Peiffer, K.-H.; Sommer, L.; Susser, S.; Vermehren, J.; Herrmann, E.; Döring, M.; Dietz, J.; Perner, D.; Berkowski, C.; Zeuzem, S.; et al. Interferon lambda 4 genotypes and resistance-associated variants in patients infected with hepatitis $C$ virus genotypes 1 and 3. Hepatology 2016, 63, 63-73. [CrossRef] [PubMed]

62. Di Maio, V.C.; Cento, V.; Lenci, I.; Aragri, M.; Rossi, P.; Barbaliscia, S.; Melis, M.; Verucchi, G.; Magni, G.F.; Teti, E.; et al. Multiclass HCV resistance to direct-acting antiviral failure in real-life patients advocates for tailored second-line therapies. Liver Int. 2017, 37, 514-528. [CrossRef] [PubMed]

63. Ramírez, C.; Gregori, J.; Buti, M.; Tabernero, D.; Camós, S.; Casillas, R.; Quer, J.; Esteban, S.; Homs, M.; Rodriguez-Frías, F. A comparative study of ultra-deep pyrosequencing and cloning to quantitatively analyze the viral quasispecies using hepatitis B virus infection as a model. Antivir. Res. 2013, 98, 273-283. [CrossRef]

(C) 2019 by the authors. Licensee MDPI, Basel, Switzerland. This article is an open access article distributed under the terms and conditions of the Creative Commons Attribution (CC BY) license (http://creativecommons.org/licenses/by/4.0/). 